

Abstract 873 Figure 2 P chart: PICU continuous infusions matching the current order group of 20

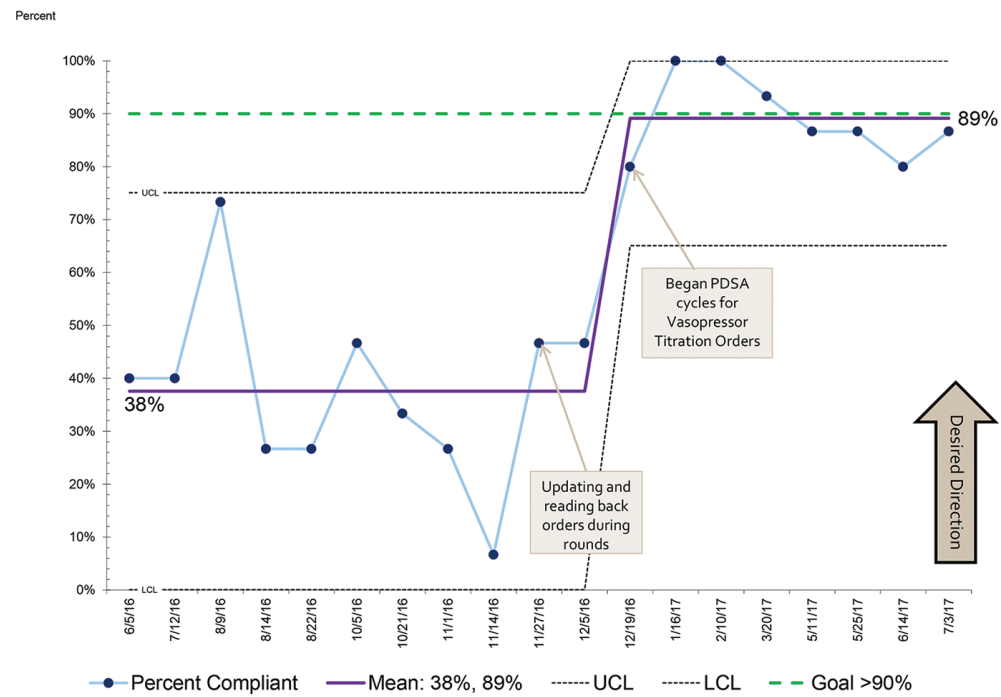

Abstract 873 Figure 3 P chart: PICU vasoactive medications matching the provider order group of 15

Conclusions Ensuring continuous medications have matching provider orders uses proven safety mechanisms, ensures nurses provide care within their scope of practice, and reduces adverse drug events. This project will be spread to the Cardiac ICU and has been used to develop guidelines for medication orders in EPIC.

\section{A QUALITY IMPROVEMENT PROGRAM TO ACHIEVE INDEPENDENCE IN HOME CENTRAL LINE CARE BY PAEDIATRIC ONCOLOGY FAMILIES}

${ }^{1}$ Chris Wong, ${ }^{2}$ Constance Barysauskas, ${ }^{3}$ Marie Desrochers, ${ }^{3}$ Margaret Brill-Conway, ${ }^{3}$ Riley Mahan, ${ }^{3}$ Kelly Eng, ${ }^{3}$ Amy Billett. ${ }^{1}$ Dana-Farber/Boston Children's Cancer and Blood Disorders Centre, US; ${ }^{2}$ Dana-Farber Cancer Institute, US; ${ }^{3}$ Blood Disorders Centre, US

\subsection{6/bmjoq-2017-IHI.7}

Background Central line associated blood stream infections (CLABSI) occur frequently in the paediatric oncology (PO) population increasing morbidity, mortality, and costs. Families in the home provide a large portion of PO external central line (CL) care with limited opportunities to develop skills that maximise best practice bundle adherence, key to infection prevention.

Objectives To achieve $>90 \%$ of families with documented independence in CL flushing by 12 months.

Methods Plan-do-study-act cycles were used to test changes from April 2016-June 2017 after identifying drivers in a pilot (June 2015-March 2016) to develop a family-centred learning curriculum. This involved return-demonstration [teach-backs (TBs)] of CL skills in front of a nurse coach during routine clinic visits and use of CL care cognitive aids. Key interventions: culture change-new expectation of $\mathrm{TB}$ participation until independent; embedding TBs into routine care with a dedicated nurse champion; developing checklists to standardise content, approach, performance, and proficiency evaluation with documentation. Statistical process control charts tracked changes over time.

Results During the pilot, $12.9 \%(22 / 171)$ participated in the TB program and $4.7 \%(n=8)$ had documented independence. After April 2016, special cause variation shifted the centre line of families' independent from $6 \%$ to $89 \%$. Participation 


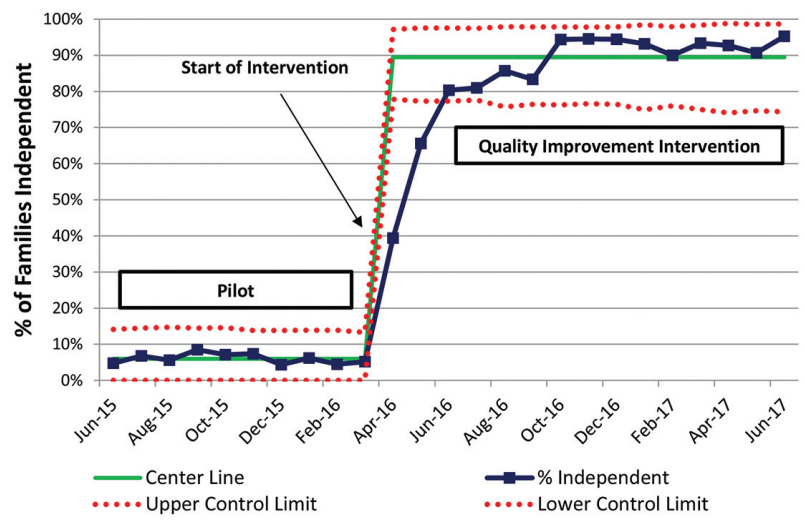

Abstract 886 Figure 1 Statistical process control chart (p-chart) 3 sigma

increased to $87.9 \%(181 / 206)$ with $82.5 \%(n=170)$ independent, sustaining the improvement at $>90 \%$ since October 2016.

Conclusions Ensuring families' independence with CL care in the home is a safety priority which can be achieved through a teach-back program embedded into routine care. Determining impact on families' CL care-associated distress and ambulatory CLABSI rates is in process.

\section{STOP MISSING ELEVATED BLOOD PRESSURES (BP): USING QI METHODOLOGY TO IMPROVE BP MANAGEMENT IN AN OUTPATIENT PAEDIATRIC NEPHROLOGY CLINIC}

${ }^{1}$ Hailey Woollen, ${ }^{2}$ Maureen Walsh Koricke, ${ }^{3}$ Cheryl Courtlandt, ${ }^{3}$ Karen Emmerton, ${ }^{3}$ Mehgan Beverley, ${ }^{3}$ Susan Massengill. ${ }^{1}$ Cincinnati Children's, US; ${ }^{2}$ Queens University of Charlotte, US; ${ }^{3}$ Levine Children's Hospital @ CMC, US

\subsection{6/bmjoq-2017-IHI.8}

Background Despite previous studies demonstrating accelerated deterioration in kidney function due to hypertension in paediatric patients with chronic kidney disease, there are significant discrepancies in the identification and management of elevated blood pressure in this high-risk population. Recognition of hypertension during childhood is hampered by the need for gender, age and height adjustment in calculations. We

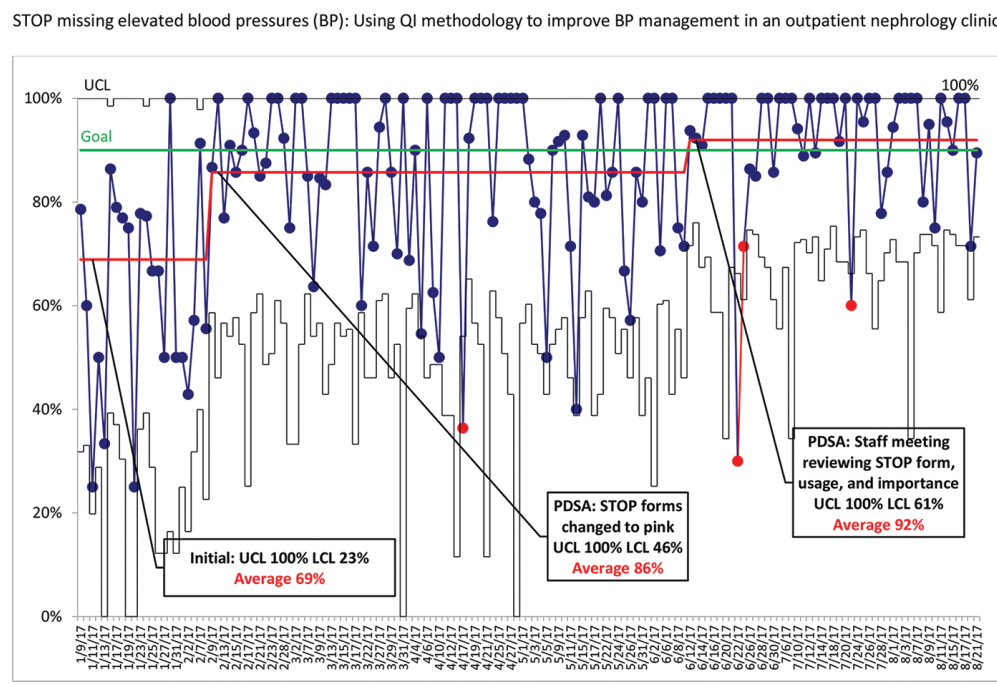

Abstract 899 Figure 1 Percentage of clinic visits with STOP forms (p chart)

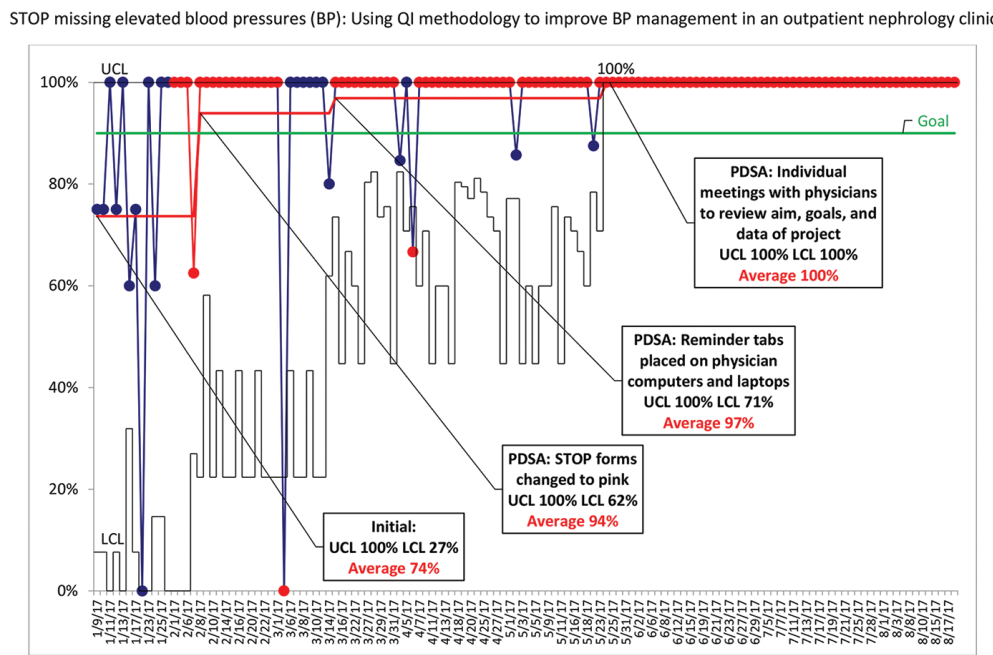

Abstract 899 Figure 2 Elevated BP percentile with intervention ( $p$ chart) 